\title{
Chondroitin sulfate proteoglycans regulate the growth, differentiation and migration of multipotential neural precursor cells through integrins signaling pathway
}

\author{
Wenli Gu ${ }^{1,2}$, Saili Fu ${ }^{1}$, Yanxia Wang ${ }^{1}$, Ying $\mathrm{Li}^{1}$, Hezuo $\mathrm{Lü}^{1}$, Peihua $\mathrm{Lu}^{1}$ \\ ${ }^{1}$ Department of Neurobiology, Shanghai Jiao Tong University School of Medicine, ${ }^{2}$ Department of Clinical Laboratory No.9th \\ People's Hospital of Shanghai Jiao Tong University, Shanghai, China
}

Neural precursor cells (NPCs) are defined by their ability to proliferate, self-renew, and retain the potential to differentiate into neurons and glia. Deciphering the factors that regulate their behaviors will greatly aid in their use as models of development and as therapeutic agents. Chondroitin sulfate proteoglycans (CSPGs) are prominent components of the extracellular matrix (ECM) in the central nervous system (CNS) and are assumed to play a particularly important role in controlling neuronal differentiation and development. In the present study, we firstly demonstrated that CSPGs were constitutively expressed on the NPCs from E16 rat embryonic brain by double immunofluorescence. And from a series of changes of cell biological property induced by abolishing the functions of endogenous CSPGs on the surface of NPCs using Chondroitinase ABC, we verified that these molecules may take a crucial part in the regulatory function of NPCs proliferation, differentiation and migration. In addition, we also provided the evidence that integrins signal pathways might be involved in the regulatory function of CSPGs on NPCs. All together, the objective of our study was to have a better understanding of the regulation roles of CSPGs on the development of NPCs and the corresponding mechanisms in the CNS, therefore supply some more useful clinical strategy for the treatment of the neurological diseases and injuries in the CNS.

Keywords: CSPGs, differentiation, growth, integrins, migration, NPCs

Cell Research (2008) 18:s139. doi: 10.1038/cr.2008.229; published online 4 August 2008

Correspondence: Peihua Lu

E-mail: peihualua3@yahoo.com.cn 